\title{
Some limits to evidence-based medicine: a case study from elective orthopaedics
}

\author{
Ewan Ferlie, Martin Wood, Louise Fitzgerald
}

\begin{abstract}
There has been growing interest in recent years in the application of the principles of evidence-based medicine (EBM), although implementation is complex. Scientific, organisational, and behavioural factors all combine to shape clinical behaviour change. Case study based qualitative data are presented which illuminate such processes within one clinical setting (elective orthopaedics), drawn from a larger study. It is suggested that (1) there are alternative models of what constitutes "evidence" in use; (2) scientific knowledge is in part socially constructed; and (3) clinical professionals retain a monopoly of technical knowledge. The implication is that there may be severe obstacles to the rapid or broad implementation of EBM. (Quality in Health Care 1999;8:99-107)
\end{abstract}

Keywords: case study; orthopaedics; evidence-based medicine

Introduction and background

There has been increasing interest in the application of the principles of evidence-based medicine (EBM) to health care, with an associated growth of meta-analysis, systematic reviews, and guideline production. Such work is likely to continue and expand given the strong focus on clinical effectiveness now apparent in UK health policy and indeed in many other healthcare systems. Clinical quality is a core objective of the new agenda, to be achieved in the UK through the introduction of new evidence-based national service frameworks and the National Institute for Clinical Effectiveness, charged with "drawing up new guidelines and ensuring they reach all parts of the health service". ${ }^{1}$

A key policy issue is therefore how to ensure full implementation in the field. This raises a set of social science questions of how work practices change - or do not change - in highly professional organisations such as health care. From a social science perspective, the experience of difficulties in research and development implementation raises fundamental issues about a possible contest between different forms of clinical knowledge. The world of evidence-based medicine can be characterised by an abstracted form of pure rationality, often of a meta-analytic nature, ${ }^{2}$ where there is a clear hierarchy of evidence at the apex of which lies the randomised controlled trial. The world of clinical practitioners, by contrast, may be much more local and experiential in nature.

A crudely linear and rationalistic model of technology transfer is also often evident, whereby knowledge flows freely from the research laboratory to the field of practice. It is true that some behavioural obstacles to implementation have been identified within the policy literature, such as the intermediate role of professional associations and opinion leaders in diffusing information. ${ }^{3}$ Within this dissemination model there is typically a search for effective levers of clinical behaviour change. More interpretive perspectives offer a stronger account of the causes of research and development non-implementation: knowledge does not flow across organisational boundaries because practice is made locally by different stakeholders who have different interests, models of practice, and views of the clinical knowledge base. ${ }^{4}$

Within organisational research the problem is investigating how decisions get made in actual healthcare organisations. Do clinicians base their practice on clear science? Under what circumstances do clinicians change their behaviour? How successful are the external interventions designed to achieve planned change in clinician behaviour? ${ }^{6}$

This paper will argue that some scepticism is needed in relation to the probable long term impact of EBM. Non-implementation or very partial implementation is likely to be the fate of many interventions based on EBM. Firstly, this is because much of the science is seen in practice as inconclusive or as contested. Secondly, it is because groups of professionals such as clinicians retain substantial autonomy over their work practices ${ }^{7}$ and resist external interventions from a generally marginal and powerless research and development function. Thirdly, it is because much clinical knowledge is tacit and experiential in nature, and thus seen as more of a craft than a science, so that the findings of EBM are not fully accepted by practitioners as valid in their practice. 
ACHIEVING CHANGE IN CLINICAL PRACTICE: SCIENTIFIC, ORGANISATIONAL, AND BEHAVIOURAL FACTORS

We have been part of a research team that has investigated empirically within one UK NHS region the impact of scientific and nonscientific factors on patterns of clinical decision making within acute sector settings. The four case studies selected were:

- The introduction of an innovative form of anti-coagulation service provision, moving across sectoral boundaries from a secondary to a primary care setting; this innovation was a pilot project which was evaluated as successful but was found difficult to diffuse

- The implementation of the "changing childbirth" document, with subsequent role renegotiation between obstetricians and midwives in the definition and treatment of "high risk" pregnancies; this was facilitated by a carefully designed national implementation strategy which scripted in the key players

- The diffusion of a technological innovation-namely, the use of laparoscopic surgery in the case of inguinal hernia; the pattern here was rapid initial diffusion in advance of strong evidence followed by a reappraisal

- The uptake of a particular novel drugnamely, the use of low molecular weight heparin (LMWH) as antithrombolytic prophlyaxis after elective orthopaedic surgery for hips and knees. This was presented as a candidate for evidence-based intervention initially. However, the case is interesting because of a continuing scientific controversy which defied easy resolution.

A final project report is now available, ${ }^{8}$ and this paper reports the results from the orthopaedics case.

\section{Methodology and research design}

QUALITATIVE RESEARCH METHODOLOGY

The research project was based on a qualitative and comparative case study design. Although this methodology cannot offer statistical generalisability, it is better suited to the problem selected than other quantitative methods, such as randomised controlled trials. ${ }^{9}$ Such case study based approaches can capture the meaning to human actors of social and organisational processes more readily than quantitative methods. They are more sensitive to the impact of local and historical contexts. This is particularly important where there is a range of different stakeholders, each advancing a different version of reality (the decision rule has been to identify and expose such variation in accounts received). Case study methodology is holistic rather than reductionist, and can thus explore how complex systems develop through time. The consideration of historical antecedents and the establishment of an analytical history are then important advantages of the methodology.

Standard objections to case study methods include their presumed lack of connection to theory (or hypotheses) and the nongeneralisable nature of the results generated.
Each of these objections can be dealt with in turn. Firstly, although data typically emerge inductively rather than deductively, as is the case in much natural science, they should be connected back to theory as the analysis becomes more advanced and thematic. In addition, early cases were selected against clear selection criteria to explore possible propositions (for example, two cases were initially presented as evidence based and two were not). Secondly, the comparative method enables us to derive early propositions and to identify patterns which can then be tested against a wider data base (perhaps using different survey methods).

\section{RESEARCH DESIGN}

The empirical research consisted of two sequential stages. In stage 1 we undertook a broad exploratory sweep of each of the four clinical change issues across the region on the basis of key names supplied by the region followed by a "snowballing" technique whereby other names emerged. In stage 2 we undertook a more intensive analysis of one clinical group dealing with this change issue. This provided a set of eight basic case studies.

Data were collected through semistructured interviews with clinicians and other stakeholders, as well as a review of publications in clinical and other journals. A core pro forma was given to all respondents by the three researchers working on the project (who each led on particular issues), although the interview also contained some customised and issue specific questions. About 130 interviews in all were conducted, tape recorded, and transcribed (27 in orthopaedics). A contents analysis was done on this material as the basis for the initial case studies.

\section{Empirical findings from the orthopaedic} cases CONTEXT AND HISTORY OF THE ISSUE

There has been a long standing debate about the risk of-and identification of optimal prophlyaxis for-deep vein thrombosis and pulmonary embolus after surgery. This debate extends across general, vascular, and orthopaedic surgery. It was initially suggested that the use of the novel drug of LMWH was now supported by evidence. Our interviews, by contrast, suggested that this remained controversial in orthopaedic surgery, especially in the case of elective surgery for knees and hips.

This research question has stimulated numerous randomised controlled trials, metaanalyses, and consensus statements. Some key thrombosis research groups have been active since the early 1970s, both in general and orthopaedic surgical settings. Kakkar's overview of this work suggests high frequencies of deep vein thrombosis after orthopaedic surgery $\left(65-75 \%\right.$ for knee replacement ${ }^{10} ; 50-55 \%$ for elective hip replacement). Pulmonary embolus event rates are rarer, but are of course serious clinically.

A succession of preventive methods has been considered, including mechanical methods (stockings and boots) and drugs (standard 
heparin; warfarin and aspirin). A new generation of LMWHs came on to the market in the 1980 s, holding out the promise of lower haemorrhagic events and easier administration as well as greater cost. They have been widely adopted in European countries, where nearly all high risk orthopaedic patients would be given LMWH.

The first prospective double blind randomised controlled trial of the efficacy of LMWHs for major abdominal surgery was published in 1982. ${ }^{11}$ Kakkar's later overview concluded that in orthopaedic surgery, "LMWH is as, or more, effective than heparin; as effective as heparin in combination with dihydroergotamine and more effective than dextran" ${ }^{10}$ Research also began on competing modalities, thus Fordyce and Ling report that the use of a venous foot pump can reduce thrombosis after total hip replacement. ${ }^{12}$

Various meta-analyses have also been produced. Collins et al conducted a meta-analysis of trials to assess the impact (against controls) of giving subcutaneous heparin as a prophlyaxis against deep vein thrombosis and pulmonary embolus. ${ }^{13}$ Although the numbers in the orthopaedic trials were not big enough to allow firm conclusions, the data were suggestive of substantial reductions in deep vein thrombosis and pulmonary embolus without substantial increase in serious bleeding. Another meta-analysis of trial evidence comparing standard heparin and LMWH concluded that there was no convincing effect in general surgery, ${ }^{14}$ although LMWH may be preferable for patients having orthopaedic surgery in view of the large deep vein thrombosis risk reduction.

Further meta-analyses confirmed that LMWHs are better than placebo in preventing deep venous thrombosis in orthopaedic and general surgical practice and better than dextran. ${ }^{15}{ }^{16}$ Initial evidence suggested that LMWHs were better than standard heparin in both general surgical and orthopaedic practice, although larger randomised controlled trials were needed. There was no indication of a significant difference in the incidence of major haemorrhages. Further support for the use of LMWH in orthopaedic surgery came from other overview studies. ${ }^{17}$

The THRIFT (Thromboembolic Risk Factors) consensus group reviewed the use of various forms of prophlyaxis for venous thrombosis embolism in different clinical settings. ${ }^{18}$ They urged clinicians to develop written policies and to audit their activity. Patients should be assessed for risk, and categorised into low and moderate/high risk groups. As far as elective hip patients were concerned, high efficacy regimens should be used (either LMWH or adjusted dose regimen of subcutaneous heparin in preference to fixed low dose heparin, adjusted dose warfarin, intermittent pneumatic compression, or dextran). Little reliable evidence existed for knees.

Recent papers have included one from the antiplatelet trials collaboration which reviewed all randomised controlled trial evidence on deep vein thrombosis. ${ }^{19}$ Preliminary data sug- gested that anti-platelet treatment, at least for pulmonary embolus, suggested more protection than from heparin alone. They again stressed the importance of orthopaedic surgeons developing an anti-thrombolytic policy.

Interestingly, an intense scientific controversy has recently re-emerged. In a controversial and widely circulated paper, Murray et al's "opportunistic" meta-analysis argued that the current pulmonary embolus event rate (as opposed to the conventional focus on deep vein thrombosis) is an order of magnitude lower than that usually quoted and therefore the potential benefit of prophlyaxis is small and may not be worth the risk. ${ }^{20}$ Consensus statements have incorporated old figures from the 1960s and they therefore are not reliable. Guidelines which recommend the routine use of any form of prophlyaxis after hip replacement are also not justified. This paper by Murray et al thus challenged what it saw as the "dogma" of routine prophylaxis.

The most recent attempt to provide guidelines has been undertaken by an expert group drawn from different branches of surgeryincluding leading orthopaedic surgeonsworking along consensus lines. ${ }^{21}$ Careful reading of the document suggests that continuing scientific controversy was evident in two areas. Firstly, group members could not agree as to the quality of evidence produced from metaanalyses (p21) as opposed to primary randomised controlled trials. Secondly, it appeared to be particularly difficult to establish clear guidelines in elective orthopaedics and further prospective audits were called for (p18) to establish clearly the mortality and incidence of fatal pulmonary embolus in this setting. Clearly, there is as yet no convergence on a clear scientific consensus; the debate continues.

ORGANISATION OF ORTHOPAEDIC WORK

Orthopaedic surgery represents a highly professionalised form of clinical work, where the national professional association (the British Orthopaedic Association) and internal journals (especially the Fournal of Bone and foint Surgery) represent powerful internal mechanisms for diffusing accredited information. The orthopaedic community was described as self contained and inward looking in nature, with a strong culture quite distinct from general or vascular surgery. The implication was that results obtained in vascular or general surgical settings were not seen as automatically applicable to orthopaedic settings: "orthopaedics is different"

Certain hospitals act as specialist orthopaedic centres of excellence, radiating out to the service departments in the local hospitals. A flourishing private sector also exists which could act to draw practitioners away from developing a research orientation. This professional community was resilient and strongly embedded, retaining effective control over a core heartland of clinical practice despite all the macro level reorganisations apparent since 1990. 
Table 1 Map of reported current practice in relation to elective orthopaedic surgery (hips and knees)

\begin{tabular}{lll}
\hline Hospital & Current practice & Recent shift \\
\hline 1 & LMWH foot pumps & Away from warfarin or standard heparin \\
2 & LMWH & Away from warfarin \\
3 & LMWH/stockings & Stable \\
4 & LMWH & Stable \\
5 & Heparin/stockings & Retreat from LMWH \\
6 & LMWH (hips) & Stable \\
7 & LMWH & Partial retreat from LMWH \\
8 & LMWH (high risk) & Review of high dosage \\
9 & LMWH & Shift to heparin \\
10 & Heparin & Stable \\
11 & Heparin & \\
\hline
\end{tabular}

When compared with general or vascular surgery, certain distinctive characteristics of orthopaedics as a clinical setting were described. Firstly, the historic research base within orthopaedics was generally undeveloped because orthopaedic surgeons appeared to be more action ("doers") than reflection orientated. In addition, research in orthopaedics was in any case complicated by the long term nature of the clinical outcomes (for example, hip replacement) which would need to be studied. Anti-coagulation prophlyaxis represented an unusual area where a large number of randomised controlled trials had taken place. However, many orthopaedic surgeons were not avid readers of academic papers and the translation of research results into practice was by no means easy.

Secondly, the personality structure of orthopaedic surgeons was seen as distinct. The respondents described themselves as clinicians who needed to be decisive in risky clinical situations and hence would behave as if they were certain despite contradictory claims and the lack of solid evidence: "we have got people who are deeply certain...they are binary people". The result could also be volatility and the periodic switching of opinions from one firm position to another. Different camps in the LMWH controversy held their different opinions with vigour.

Thirdly, the separate and autonomous nature of orthopaedic practice was important, and practitioners were suspicious of work done outside the discipline. The fournal of Bone and foint Surgery, with its own editorial board and referee system, was consistently held up as the most important place to publish. Interactions with general and vascular surgeons were sometimes seen as problematic, given turf disputes. Orthopaedics held distinctive norms so that, for example, aversion to and fear of bleeding was as great as the fear of deep vein thrombosis and pulmonary embolus. Disputes over prophlyaxis could sometimes become tangled up with wider negotiations with the general or vascular surgeons.

STAGE 1 RESULTS: OVERVIEW ACROSS THE REGION In Stage 1, 17 interviews were conducted with leading orthopaedic surgeons and research scientists. This produced a map of reported practice across the hospitals visited. Although these data are only small scale, nevertheless they suggest some interesting findings.
Firstly, table 1 suggests that some form of prophylaxis was evident in all sites visited. This is of interest as Brenkel and Clancy had suggested that prophylactic drugs were given by less than half of Britain's orthopaedic surgeons and that they were not convinced of their utility. ${ }^{22}$ However, a later survey found that 90 per cent of respondents reported the use of some form of prophylaxis, usually of a chemical nature. ${ }^{23}$ This survey was done just before the introduction of $\mathrm{LMWH}$, with low dose heparin emerging as the most popular drug, followed by warfarin and dextran. Many surgeons also used a combination of drugs. These current data suggest that the pattern of widespread phrophlyaxis has continued, with LMWH emerging as a popular drug.

Provisional change outcome: rapid change, but volatile and contested

Table 1 confirms that the question of prophylaxis policy has now been generally addressed at some level by many orthopaedic surgeons. They have of course been urged to do so by consensus statements and some research findings. One such article which covered various surgical settings concluded, "it is believed that the emphasis is now on the clinicians who must protect all high risk patients with $\mathrm{LMWH}$ prophylaxis". ${ }^{24}$

Although there was a range of different modalities reported, LMWH emerged as the single most popular. This study therefore suggests rapid diffusion of LMWH in the past five or six years and hence-in our termsthere was evidence of relatively rapid clinical behaviour change. A wide range of different modalities was still reported, however, and in three of the 11 sites there was evidence of a measured retreat in the use of LMWH after its initial adoption. The key adoption decisions were made at individual consultant or orthopaedic group level, and higher tier bodies (such as regional committees) were not seen as important. In a few sites, enthusiasts were moving to the use of mechanical devices such as foot pumps and away from chemical agents. So there is no convergence on a consensus of practice, and very different views were apparent. The research judgment about the change outcome observed is therefore provisional in nature and may change along with patterns of practice if, for example, there were to be a full scale retreat from the use of LMWH.

\section{Active scientific controversy and issue} intractability

"There are camps. And they are well defined camps in very entrenched positions."

The field was characterised not by movement to an agreed scientific consensus as more data accumulated but rather by the periodic flaring up of active controversy, at least between different researchers and research groups who acted as the primary producers of scientific knowledge. This pattern of continuing scientific controversy was again evident in 1996, with a questioning of reported pulmonary embolus event rate data ${ }^{20}$ on the grounds that they were badly dated and that practice 
had subsequently improved. This controversy in turn made the production of consensus guidelines difficult. ${ }^{21}$ The controversy was of a highly technical nature based on the interpretation of research design and results and so difficult for many practitioners to follow directly.

The result was that the issue was seen as intractable and perhaps even as incapable of resolution. Research had been ongoing since the 1970s, but it was reported by respondents that "there is nothing convincing out there". There were periodic flurries as research groups reopened the controversy, but many practitioners in service departments were more interested in devising a pragmatic policy to ensure that they would be seen to have taken prophylaxis seriously.

\section{Randomised controlled trials and other forms of evidence}

Practising clinicians reported differing views about the status and use of formal scientific evidence. There was a widely reportedalthough by no means universal-commitment to idealised randomised controlled trials as "the gold standard" of evidence. This was often coupled with the view that the results from actual randomised controlled trials in orthopaedics were always equivocal and done on too small a patient base. There was also suspicion of trials that had been conducted with general or vascular surgical patients as well as orthopaedic patients. Despite the long tradition of randomised controlled trials work, it was thus seen as inconclusive. For example, one respondent argued, "there is no trial in existence that shows absolutely that the use of these has any effect on the mortality rate ... .a lot of the tests are flawed in the way they have been performed". Many respondents wanted to follow the science, but were puzzled that it was so ambiguous and did not always seem to concord with current practice (for example, the use of outdated data which no longer reflected current conditions). The problem was that a paper was available to support every modality or position on the basis of selective reading. For example:

"This Fordyce and Wing paper has tipped me in the direction of foot pumps. I think that is a prospective randomised study which is well controlled. You could argue that they did not have proper control groups and they could have compared it with klexane and warfarin. But you have to start somewhere."

There were also important indirect channels of information transmission at work. These would include the British Orthopaedics Association, professional conferences and opinion leaders, and the professional journal, The fournal of Bone and Foint Surgery. Consensus statements which included leading members of the orthopaedic community (such as the THRIFT group) were also seen as important. Key decisions about modality adoption were made locally within orthopaedic groups, largely as a result of negotiation among the consultant colleagues. Historic links between these groups and local teaching hospitals were also impor- tant in the diffusion of practice and received wisdom.

Another view was that long clinical experience was more important than formal science and enabled the practitioner to recognise local clusters and patterns:

"I am more inclined to the clinical side. Because I don't think it really matters what happens in the lab; it is what happens to the patient that matters. And that to me is much more convincing."

These clinicians were far more sceptical about the possible contribution of formal scientific knowledge to practice, and regarded orthopaedic surgery as much as a learnt craft as it is a science. Tacit and experiential forms of knowledge were here seen as more important than formal scientific knowledge.

\section{Role of big science}

Although orthopaedic surgeons represented the key body of actors, there were also a few big and well established research units in the field undertaking large scale randomised controlled trials. Some of these senior researchers were of international standing and were influential in the field. Their reputations were such that their papers were more likely to be read and believed. These units were important, especially as research fellows and registrars rotated out of the research units and into the service departments. Nevertheless, the link between the research units and the service departments remained partial and indeed difficult in places.

Non-scientific influences on decision making Various other non-scientific factors were also mentioned as important influences on decision making in this field:

- Pharmaceutical companies: the new generation of mini heparins was, of course, under patent and marketed by the pharmaceutical companies. Although direct approaches by drug representatives were not seen as effective, more indirect and subtle forms of marketing included sponsoring research, professional conferences, and well known speakers drawn from the orthopaedic community. One respondent reported: "It was only as a result of drug company publicity that we heard of the LWMHs. There was a great deal of drug company money thrown about at the time. There were free lunches. The whole drug company marketing effort went on getting us to use these rather expensive chemicals on a regular or universal basis."

- Medicolegal considerations. There was anxiety about possible accusations of negligence following any pulmonary embolus. Although no cases have come to court as yet, some are being settled out of court. The view expressed in print by some eminent clinicians was that it would be negligent to have done nothing, although if some form of precaution had been adopted that would be a defence.

- Ease of administration: the development of a group policy was sometimes undertaken on pragmatic grounds that it would make administration of drugs easier for nursing 
and junior medical staff than if every consultant operated a different regimen:

"I tend to give klexane, not because I am convinced that it works, but because it is easier practice just to say 'all the joints to have klexane,"

- Individual patient deaths: many respondents could vividly recall deaths of patients either from pulmonary embolus or indeed from haemotomas, and this personal experience could cause clinicians to review their practices.

Many other factors were not generally seen as important in decision making. These included the higher tiers of the healthcare system; the research and development function; healthcare purchasers (local health authorities who were busy downsizing and were concentrating on cost and volume rather than more difficult clinical effectiveness issues, and family doctors with devolved budgets who had in general a weak grasp of research literature); general managers; nurses; and pharmacy and patient groups (patients were grateful for what were generally successful operations). The picture was painted of an issue largely processed by the core professional grouping of orthopaedic surgeons if only because few others had technical expertise or legitimacy. Initiatives emanating from outside this core group were unlikely to be believed. For example, some respondents reported a distrust of meta-analyses on the grounds that, "they are done by statisticians who have little knowledge of the clinical side of it”.

STAGE 2 RESULTS: INTENSIVE ANALYSIS OF ONE GROUP'S POLICY MAKING

In stage 2 , an intensive analysis of policy making (10 further interviews) was undertaken within one orthopaedic group (hospital number 8 in table 1) where initial interviews indicated a recent partial retreat in the use of LMWH. So how was this decision taken? How was it implemented across the group?

\section{Context and background}

This was a large and well established group of some 5.5 consultants set in a local hospital. There were also many junior medical staff, with rotation links into teaching hospitals. There is a 24 bedded ward, busy outpatient clinics, and also a flourishing private sector. Within the hospital, workload has built up and is now estimated at 200 elective knee operations each year and 150 hip operations. There is a strong service orientation, with the emphasis on fast throughput and hitting activity targets rather than basic research. Indeed, this was seen as a distraction according to one respondent because "everyone is worried that their careers could be messed up by pure research". The group is not participating in multicentre trials, although some applied research is going on.

The core group of consultants remains the key decision making body. Interpersonal relations are good, with a pattern of informal specialisation but also a low threshold for cross referral. In addition, various mechanisms exist to ensure effective within group communication. There was a daily trauma list meeting, as well as an audit meeting and orthopaedic cog meetings every two months, followed by an informal dinner of all the consultants where many of the real decisions were made.

The orthopaedic group is regarded as clinically excellent, with a strong emphasis on continuing professional development and participation in professional bodies. The group is well organised, with a tradition of budgetary control and of winning contracts for the hospital. Relations with local general managers are good, but are poorer with the hospital's corporate centre. There is strong group identity and cohesion, although the orthopaedics group was also seen as inward looking by outsiders at times. There is a part time clinical director role (held by a younger consultant), but also a head of service role (held by the most senior consultant). The clinical director was perceived as undertaking an administrative burden but not as exercising any managerial authority over the other consultants. The form of decision making thus remained highly collegial, with a norm of gentlemanly behaviour and a search for consensus within the group.

The hospital has recently launched a corporate clinical effectiveness group, on which the orthopaedic surgeons are represented, charged with getting evidence into practice. It will be interesting to follow the degree to which this corporate initiative manages to achieve planned change within the clinical groups. So far it has not addressed the question of pulmonary embolus in elective orthopaedics, and its early priorities lay elsewhere.

\section{Development of the first set of guidelines}

Two of the younger consultants (one of whom is also clinical director) concentrate on the bulk of elective knees and hips, although the others do small numbers. They are seen as the informal leaders on this issue by the rest of the group, just as the other consultants would legitimately lead on "their" issues. The other consultants have all built up their own subfields, such as spine surgery, where they have special expertise. A third consultant had some time ago a research interest in $\mathrm{LMWH}$ and so his views also carry weight in consensus formation, particularly as his research experience led him to be somewhat critical of the widespread use of klexane.

About five years ago, and triggered by the arrival of one of the younger consultants, it was felt that there was a need to develop a coherent policy for thrombolytic prevention given growing concern nationally about the risk of pulmonary embolus. This was driven in part by a fear of possible medicolegal intervention and the subsequent need to have a coherent policy in place. Guideline construction progressed on an informal and collective basis, building up discussions from the two consultants most actively involved to the rest of the group. The professional literature informally "filtered in" to the group through their previous reading and understanding. An initial set of guidelines (that is, not protocols) was then drawn up and 
agreed verbally (not written down), indicating the widespread use of klexane. One of the leading consultants had seen problems with "soggy wounds" elsewhere with the use of warfarin, and so was keen to try a different drug and klexane seemed a good buy at the time. Here is one description of the process by one of the leading consultants:

"Initially it was just me, but then as I was leading the joint replacement group at that stage, it got adopted across the whole group, by a combination of osmosis and the stuff that was written in the literature that other people read."

New problems in turn emerged, with some concern about the number of bleeds and wound haemotomas locally after the use of LMWH.

\section{Later revision of guidelines}

About three years ago, there was a revision of the guidelines, in essence away from the routine use of LMWH for certain low risk procedures (for example unilateral knees). This revision exercise was again led by the two consultants with a special interest, on the basis of their local clinical experience of haemotomas, a (selective) reading of publications, and attendance at the British Orthopaedics Association conference:

"We made a distinction between the single and the bilateral knees. There was some feeling that we were not seeing many thrombo problems in the single knees, and then at the British Orthopaedics Association conference there were several quite good papers, which are published in the proceedings of the fournal of Bone and foint Surgery, and that combined with the controversy in the BMF made us less protagonistic..."

Nevertheless, the guidelines still indicated the use of LMWH for "high risk" patients, begging the question of risk definition. It is interesting to note the focus on haemotomas as a highly visible disaster, rather than deep vein thrombosis or post discharge pulmonary embolus which may not be so apparent to orthopaedic surgeons. There was a strong belief, based on local clinical experience, that the wound healing was not as good if the patients were on LMWH.

\section{Problem of implementation}

"We sat down and discussed this subject endlessly and do have loose guidelines. We have informal guidelines rather than rigidly imposed guidelines, this is not a rigidly imposed policy so that any consultant who wishes to opt out for any given patient, we do not have a problem with that."

The bulk of the knee and hip operations are done by the two consultants who helped to draw up both sets of guidelines, thus there is no problem about ownership in their cases. A few operations were also done by the four other consultants in the group. In general, no major concerns were expressed about acceptance of informal guidelines, but there would have been resistance to the imposition of formal protocols. Some had no strong feelings and were willing to accept the guidelines so as to achieve uniformity of practice or because they fitted with their training. However, there was a difference expressed by some other consultants in the definition of "at risk". Should all hip patients be seen as at risk, or only those with a particular history? Was evidence of a considered approach to pulmonary embolus prevention enough, irrespective of the final modality selected? One consultant stated that, "my understanding is that we all accept that no prophylaxis is negligent for total joint replacement".

The form of prophylaxis could legitimately vary, depending on the practice of the individual consultant. The six consultants could perhaps be placed into three groups: two were keen on LMWH; two were neutral; and two had slight reservations about widespread use (including the only consultant with direct research experience in the field). Guidelines had to be loose enough to preserve group consensus under conditions where individuals retained autonomy over their clinical practice.

\section{Analysis and wider implications}

ANALYSIS

A major feature of the case study is the continuing high level of group and individual autonomy over work practices. The key decisions were made by the core group of consultant orthopaedic surgeons, who were seen as having the technical knowledge and also legitimacy required. Policy formation proceeded in a loose and negotiative way because it was important to build a consensus among colleagues. Even the clinical director acted as no more than an administrative coordinator, and could not-indeed did not wish to-impose a "party line" on colleagues. The corporate clinical effectiveness unit had not yet made any foray into this area, although it was still early days. General managers, patients, and nurses were all conspicuous by their absence, as were general practitioners even when they were purchasing care. On occasions when there were cases of pulmonary embolus, nurses would informally contact managers with an interest in quality in case of a complaint, but this network was disengaged from the core clinical grouping. It was also reported by some respondents that the local health authority had at one stage tried to set up a formal audit of pulmonary embolus, but it was difficult to agree this with the orthopaedic group.

Secondly, a spectrum of views about the status of formal science was apparent. Some consultants insisted that randomised controlled trials remained the gold standard, although the evidence in practice was inconclusive. Group knowledge of randomised controlled trials came from reading papers by eminent researchers in major journals and attendance at professional association meetings. However, some other (perhaps the more experienced) consultants advanced a quite different model of knowledge, based on tacit and experiential craft knowledge. Practice was learnt slowly and does not transfer from one group to another or even from one individual to another:

"If you go by the published word, it may not necessarily work in your hands because the various facets of your practice may not relate to what hap- 
pens in other people's practices. In my own field, what works for me may not work for other people. So I am much more interested in my latest bad results than I am by a string of papers from America."

A reflective practitioner should examine his own practice and identify local patterns or clusters through active self audit or peer audit, particularly where there are grounds for concern, and take corrective action. This leads to an alternative good practice model of development with a strong emphasis on continuing professional development and individual learning and reflection rather than the use of formal EBM principles.

Thirdly, several important non-scientific influences over decision making were apparent within the case study. These included the fear of possible medical-legal intervention; the need to ensure ease of administration of the drug across the clinical group; the successful marketing of a new drug by drug companies; imitative behaviour as a critical mass of colleagues adopts a new modality; and unhappy experience with individual patients which creates a counter reaction. Science is but one of a range of factors which impinge on patterns of clinical behaviour change.

POSSIBLE WIDER IMPLICATIONS

This paper reports results from small scale case study work, but many of the themes are apparent in the other case studies in our project. ${ }^{8}$ Other studies of health care undertaken by different research groups suggest similar findings ${ }^{2}$ in relation to the co-existence of different and sometimes antagonistic worlds within the overall research and development field. Knowledge flows with difficulty across these organisational, occupational, and cultural barriers. Further empirical results are needed to validate - or disconfirm - the emerging proposition that there are these fundamental organisational and behavioural barriers to rapid implementation of research and development results. This would indicate a reappraisal going well beyond a search for more effective levers of planned change.

If this is confirmed empirically, then there are implications for research and development policy in health care. It would suggest that the prospect of major "pay off” for EBM interventions might be more confined than sometimes asssumed. It would indicate that there might be other ways of improving clinical quality, such as the use of a continuing professional development model. It would question the viability of external interventions (for example from the research and development function) which are not owned by the key professional groups and associations themselves. The development phase of the research and development cycle may be as important and as problematic in its own right as the research phase where effort is currently concentrated.

Theoretically, these findings are consistent with many classic sociological analyses of how decisions are made in highly professionalised organisations such as hospitals. ${ }^{25}{ }^{26}$ An elite of professional workers secures a high degree of autonomy for itself on the basis of possession of technical expertise, public legitimacy, and control over the labour market. Ever more technical subfields emerge as knowledge accumulates, which are difficult for lay personnel to understand. Within the core group there is stress on collegiality, but this is combined with a hierarchical relationship with other nonprofessional personnel. Although doctors may now experience more controls at a strategic level, at the level of clinical practice little seems to have changed. The implication is that practice change has to be led by the professional groupings themselves and cannot be imposed from outside. On the other hand, professional bodies should also show that they are taking an active role in self regulation, and tackle problems of non-performance. The imposition of "rules", protocols, or standards from outside managers will be unlikely to be accepted in the long term. The question in relation to the problem of research and development implementation within professional healthcare organisations is: if we knew all this, why did we appear to forget it?

The support of the organisation and management group of the NHS Executive North Thames Research and Development Directorate is acknowledged.

1 Department of Health. The new NHS: modern, dependable. London: HMSO, 1997.

2 Dawson S. Inhabiting different worlds: how can research relate to practice? Quality in Health Care 1997;6:177-9.

3 Haines A, Jones R. Implementing the findings of research. BMF 1994;308:1488-92.

4 Dawson S. Never mind solutions: what are the issues? Lessons of industrial technology transfer for quality in health sons of industrial technology transfer for qualt
care. Quality in Health Care 1995;4:197-203.

5 Williams F, Gibson DV, editors. Technology transfer: a communications perspective. London: Sage, 1990

6 Oxman A. No magic bullets: a systematic review of interventions designed to improve the performance of health care professionals. London: North East Thames Regional Health Authority Research and Development Directorate, 1994

7 Freidson, E. Professional powers. London: University of Chicago Press, 1986.

8 Wood M, Ferlie E, FitzGerald L. Achieving change in clinical practice: scientific, organisational, and behavioural processes. Corporate Strategy and Change, 1997.

9 Yin RK. Case study research: design and methods. Beverly Hills, CA: Sage, 1989.

10 Kakkar VV. Prevention and management of venous thrombosis. British Medical Bulletin 1994;50:871-903.

11 Kakkar VV, Djazaeri B, Fok J, et al. Low molecular weight heparin in the prophylaxis of venous thrombosis. BMF 1982;284:375-9.

12 Fordyce MJF, Ling RSM. A venous foot pump reduces thrombosis after total hip replacement. F Bone foint Surg Br 1992;74B: $45-9$.

3 Collins R, Scrimgeour A, Yusuf S, et al. Reduction in fatal pulmonary embolism and venous thrombosis by perioperative administration of subcutaneous heparin. New Engl f Med 1988;318:1162-73.

14 Nurmohamed MT, Rosendaal FR, Buller HR, et al. The efficacy and safety of low molecular weight heparin versus standard heparin in general and orthopaedic surgery: a meta-analysis. Lancet 1992;340:152-6.

15 Leizorovicz A, Haugh MC, Chapuis Samana MM, et al. Low molecular weight heparin in the prevention of perioperative thrombosis. BMf 1992;305:913-20.

16 Leizorovicz A, Simmoneau G, Decousus H, et al. Comparison of the efficacy and safety of low molecular weight heparin and unfractionated heparin in initial treatment of deep venous thrombosis: a meta-analysis. BMF 1994;309: 299-303

17 Chesterman $\mathrm{CN}$, Chong $\mathrm{BH}$. Uses of heparin. BMf 1993;306:871-2.

18 THRIFT Consensus Group. Risk of and prophylaxis for venous thrombo embolism in hospital patients. BMF 1992; 305:567-74

19 Antiplatelet Trials Collaboration. Collaborative overview of randomised trials of anti platelet therapy: III reduction in venous thrombosis and pulmonary embolism by antiplatelet prophylaxis among surgical and medical patients. BMF 1994;308:235-46.

20 Murray DW, Britton AR, Bulstrode CJK Thromboprophlyaxis and death after total hip replacement. $f$ Bone foint Surg Br 1996;78B:863-70. 
21 International Consensus Statement. Prevention of venous thrombo embolism. London: Med Orion, 1997.

22 Brenkel IJ, Clancy MJ. Total hip replacement and anti thrombolytic prophylaxis. Br f Hosp Med 1989;42:282-4.

23 Laverick MD, Croal SA, Mollan RAB. Orthopaedic surgeon and thrombo prophylaxis. BMF 1991;303:549-50.

24 Kakkar VV. Prevention of fatal pulmonary embolism. In:
Morpurgo M, editor. Pulmonary embolism. New York: Marcel Dekker, 1994.

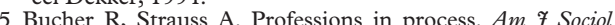
1961;66:325-34.

26 Freidson E. The centrality of professionalism to health care. In: Professionalism reborn: theory, prophecy and policy. Cambridge: Polity Press, 1994:184-98.

\section{Annual Scientific Meeting of Society for Social Medicine}

Sheffield, 8-10 September 1999

Contact for further details: Michelle Hassall, Medical Care Research Unit, SCHARR, Regent Court, 30 Regent's Streeet, Sheffield S1 4DA. Tel: 0114222 0755. Fax: 0114222 0749. Email: e.m.hassall@sheffield.ac.uk 